\title{
Sangramento espontâneo de artéria lombar em paciente com doença de Von Recklinghausen: tratamento endovascular
}

\author{
Spontaneous lumbar artery bleeding in patient with Von \\ Recklinghausen's disease: endovascular treatment
}

\section{Felipe Nasser, Breno Boueri Affonso, Charles Edouard Zurstrassen, Wilson de Oliveira Sousa Junior, Joaquim Mauricio da Motta Leal Filho, Fabio Yamada, Paulo Henrique Petterle, Francisco Cesar Carnevale*}

\section{Resumo}

Os sangramentos no retroperitônio são, em sua grande maioria, secundários a eventos traumáticos envolvendo grande energia cinética, com poucos relatos na literatura caracterizados como espontâneos. No presente relato, descrevemos paciente gestante, portadora de doença de Von Recklinghausen e com volumoso hematoma retroperitoneal diagnosticado durante o parto cesariano, secundário a ruptura espontânea de artéria lombar. A doença de Von Recklinghausen apresenta manifestações vasculares bem descritas, caracterizando-se principalmente por estenoses que são secundárias a tumores intramurais (proliferação das células de Schwann) e raramente dilatações aneurismáticas, assintomáticas em sua maioria. No presente caso, foi realizada a aortografia com cateterização seletiva e embolização da artéria sangrante com sucesso.

Palavras-chave: Hematoma, doença de Von Recklinghausen, artéria, embolização terapêutica.

\section{Introdução}

Os sangramentos retroperitoneais a partir de artérias lombares são pouco freqüentes e, em sua grande maioria, relacionados a traumas ${ }^{1,2}$ envolvendo grande quantidade de energia cinética com lesões associadas de pelve e/ou coluna vertebral ${ }^{1}$. Os sangramentos espontâneos dessas artérias são raros e com poucos relatos na literatura de língua inglesa ${ }^{1}$.

Dentre as causas de sangramentos não relacionados aos traumas, geralmente encontram-se alterações estruturais da parede arterial (aneurismas ou pseudoaneurismas) $)^{3}$ ou utilização de terapia anticoagulante . $^{4}$

\section{Abstract}

Retroperitoneal bleeding is mainly due to traumatic events with a high amount of kinetic energy, with few reported cases of spontaneous events in the literature. We report on a case of a pregnant woman with Von Recklinghausen's disease and bulky retroperitoneal hematoma diagnosed during cesarean delivery secondary to spontaneous lumbar artery rupture. Von Recklinghausen's disease has well-described vascular manifestations, mainly characterized by stenoses related to intramural tumors (Schwann cell proliferation) and rarely asymptomatic aneurysmal dilatations. In this case, aortography was performed with successful selective catheterization and embolization of the bleeding artery.

Keywords: Hematoma, von Recklinghausen's disease, artery, therapeutic embolization.

Nesse grupo, encontram-se os pacientes com doença de Von Recklinghausen, uma doença autossômica dominante, cuja incidência é de 1:3.000 e na qual o acometimento arterial é bem conhecido, porém infreqüente. As lesões estenóticas são as mais presentes, principalmente nas artérias renais, enquanto as degenerações aneurismáticas são menos freqüentes ${ }^{5}$.

Neste relato, a paciente era portadora de doença de Von Recklinghausen e apresentou um volumoso hematoma retroperitoneal causado por sangramento espontâneo de artéria lombar. Foi submetida a tratamento endovascular com embolização de micromolas com sucesso.

* Serviço de Radiologia Intervencionista e Cirurgia Endovascular, Hospital das Clínicas, Faculdade de Medicina, Universidade de São Paulo (USP), São Paulo, SP.

Não foram declarados conflitos de interesse associados à publicação deste artigo.

Artigo submetido em 20.07.08, aceito em 14.10.08

J Vasc Bras. 2008;7(4):389-392.

๑) 2008 by Sociedade Brasileira de Angiologia e de Cirurgia Vascular 


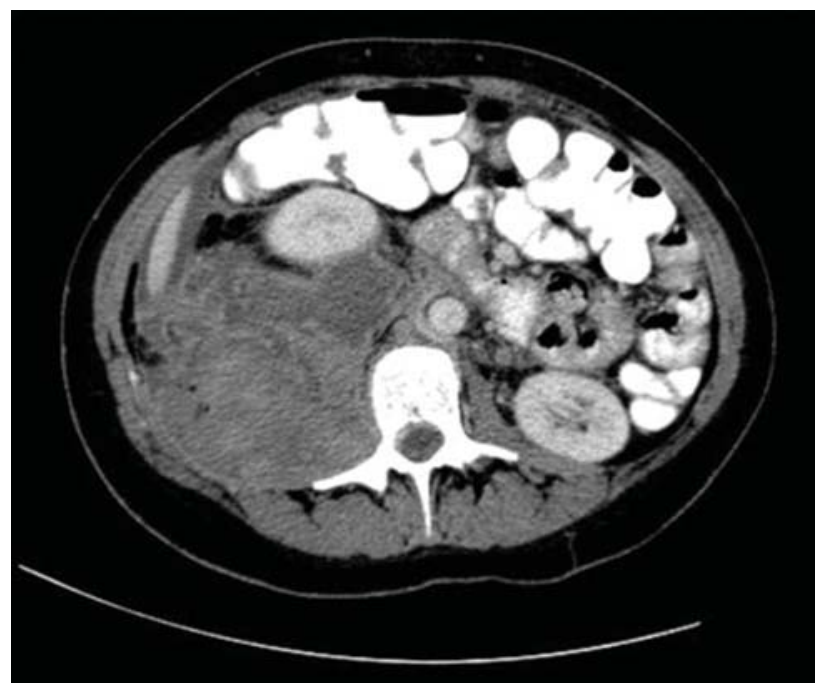

Figura 1 - Hematoma de retroperitônio secundário a sangramento da artéria lombar

\section{Relato do caso}

Paciente feminino, 36 anos, portadora de neurofibromatose tipo I e gestante com 29 semanas foi admitida no pronto-socorro com pico hipertensivo (pressão arterial: 180/110 $\mathrm{mmHg}$ ), sendo internada para controle e avaliação de vitalidade fetal. No quarto dia de internação, passou a apresentar dor lombar direita intensa com queda dos níveis de hemoglobina de 11,9 para $7,2 \mathrm{~g} \%$ associada à bradicardia fetal, sendo firmado o diagnóstico de descolamento prematuro de placenta com indicação de interrupção imediata da gestação. Durante a cesárea, foi observado grande hematoma retroperitoneal à direita. Após avaliação intra-operatória pela equipe da cirurgia geral e vascular, e não havendo sinais de expansão ou instabilidade hemodinâmica, foi tomada uma conduta conservadora, sendo indicada angiotomografia no pós-operatório imediato.

A tomografia de abdome realizada imediatamente após o procedimento cirúrgico confirmou volumoso hematoma de retroperitônio que deslocava inferior e anteriormente o rim direito e pâncreas (Figura 1), com sinal de sangramento ativo compatível com pseudoaneurisma de artéria lombar.

Foi solicitada avaliação da radiologia intervencionista, sendo realizada aortografia abdominal com punção femoral direita com introdutor 5 Fr cateter cobra 5 Fr com seletivação das artérias lombares à direita, sendo

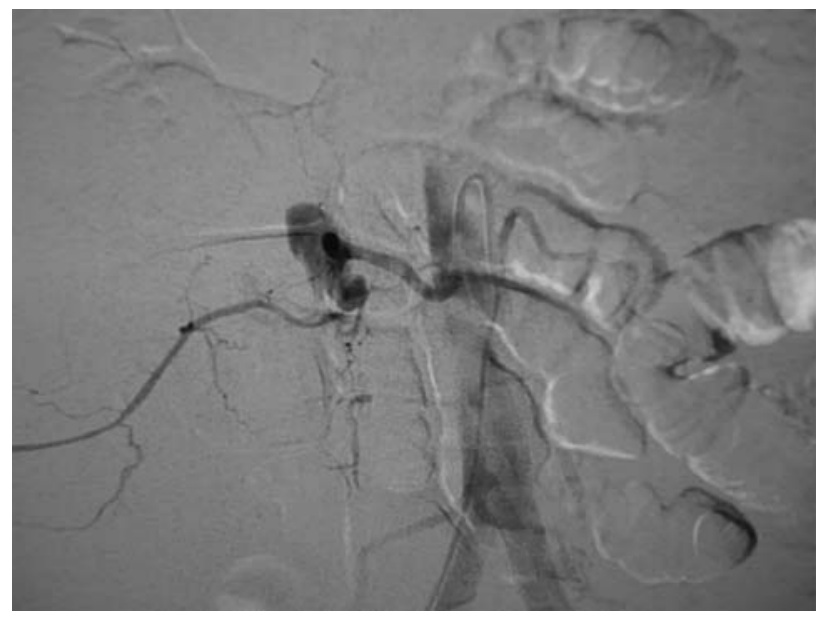

Figura 2 - Angiografia seletiva da artéria lombar com sangramento ativo

localizada a origem do sangramento a partir da segunda artéria lombar (Figura 2). Progrediu-se o microguia $(0,014$ ") e microcateter e realizou-se embolização superseletiva com micromolas. À angiografia, foi observado controle do sangramento sem extravasamentos de contraste e oclusão do ramo correspondente (Figura 3).

A paciente evoluiu sem intercorrências, tendo recebido transfusão de duas unidades de concentrados de hemácias durante a cesárea com alta hospitalar no $13^{\circ}$ dia pós-operatório. Atualmente, a paciente está sem novos sangramentos e encontra-se bem após 12 meses de seguimento.

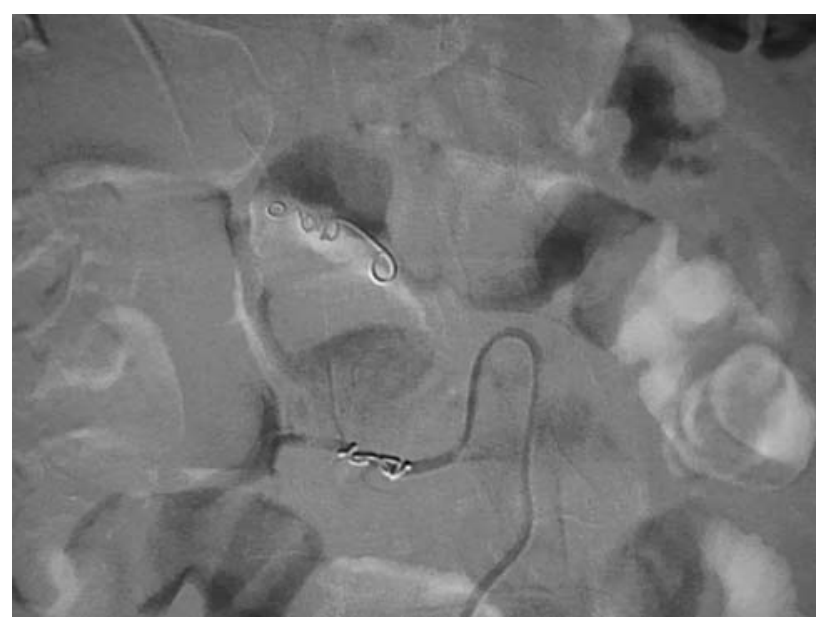

Figura 3 - Embolização superseletiva de artéria lombar com micromolas 


\section{Discussão}

Os sangramentos no retroperitônio resultantes das artérias lombares estão mais freqüentemente associados a eventos traumáticos de grande energia cinética, instrumentação cirúrgica da coluna ou, ainda, biópsias de vértebras ${ }^{6,7}$. Por outro lado, os sangramentos espontâneos dessas artérias são raros e estão relacionados à terapia anticoagulante ou mesmo ruptura de aneurismas ${ }^{8}$.

No presente relato, a paciente apresentava volumoso hematoma retroperitoneal e, na aortografia, foi evidenciado sangramento a partir de uma artéria lombar com sangramento ativo sem que se observasse dilatação aneurismática verdadeira, apesar da concomitância do diagnóstico de neurofibromatose. A neurofibromatose compreende um grupo de três disfunções genéticas caracterizadas por tumores de bainha neural $^{1,3,9}$ : neurofibromatose tipo I, tipo II e schwannomatose, que diferem entre si pelas mutações envolvidas e manifestações clinicas. A neurofibromatose tipo I (doença de Von Recklinghausen), inicialmente descrita em 1882 pelo patologista Friedrich Von Recklinghausen, caracteriza-se por sinais e sintomas variados, secundários ao acometimento dos mais diversos órgãos, desde pele com a clássica pigmentação denominada café-au-lait macule, membros, tronco com tumores de origem neuronal (neurofibromas) e sistema nervoso central (gliomas) ${ }^{5}$. As manifestações clínicas vasculares, encontradas em apenas 3,6\% dos casos, devem-se basicamente ao acometimento do leito arte$\mathrm{rial}^{5}$, sob a forma de estenoses arteriais secundárias a espessamentos intramurais (proliferação das células de Schwann); mais raramente, podem apresentar dilatações aneurismáticas, que são dilatações pósestenóticas ${ }^{10}$. Em ordem de freqüência, a artéria renal é o sítio mais acometido, porém com relatos acometendo artérias de membros inferiores ${ }^{5,10}$, artérias intercostais ${ }^{10}$ e viscerais ${ }^{11}$. Acredita-se que todos os pacientes com a doença, em avaliação post-mortem, apresentem algum grau de espessamento na parede arterial com estenoses e dilatações pós-estenóticas, porém silenciosas na grande maioria das vezes ${ }^{11}$.

A região retroperitoneal, por ser irrigada por diversos ramos arteriais ${ }^{2}$, apresenta-se desafiadora para uma abordagem cirúrgica aberta, já que uma única artéria lombar pode se comunicar com vários outros ramos arteriais, como intercostais ou ramos das artérias ilíacas internas, e que podem manter o sangramento ativo. Sclafani et al. ${ }^{12}$ relataram como múltiplas colaterais poderiam suprir um mesmo sítio de sangramento arterial $^{12}$. Os pares de artérias lombares (L1 - L4) originam-se da superfície posterior da aorta ao nível dos processos transversos, sendo que a quinta artéria lombar emerge a partir da artéria sacral lateral, da ileolombar ou, mais raramente, da artéria sacral mediana ${ }^{2}$. Todas seguem ao longo da vértebra antes de se bifurcar em seus ramos anterior e posterior na borda medial do músculo psoas, que realizam a irrigação da coluna e musculatura do dorso, respectivamente ${ }^{3}$.

Outro conceito importante é a origem da artéria radiculomedular anterior (T8 - L2) ou artéria de Adamkiewicz, que é responsável pela irrigação da porção toracolombar da medula espinhal. A manipulação inadvertida ocasionaria infarto medular e paralisia. Em cerca de $19 \%$ das vezes, a sua origem ocorre na primeira ou segunda artéria lombar ${ }^{13}$, dado que deve ser lembrado durante a manipulação e terapêutica, sempre evitando a embolização a partir da origem da artéria lombar.

Baseado nesses dados, a terapêutica deve se iniciar com aortografia e posterior seletivação de todos os ramos na área sangrante ${ }^{2}$, ao identificar o foco de sangramento, proceder com embolização superseletiva, utilizando-se microguias e microcateteres. Ao fim do procedimento, realiza-se arteriografia de controle para documentação da interrupção do sangramento ativo, bem como identificação de ramos que levem ao reenchimento dos sítios tratados.

Diante do exposto, a abordagem endovascular apresenta-se como uma opção terapêutica minimamente invasiva e com vantagens significativas sobre a abordagem cirúrgica convencional: evita-se a anestesia geral em pacientes com sangramento e instabilidade hemodinâmica; reduz a perda sanguínea provocada pelo trauma cirúrgico e dissecção do retroperitônio; oclusão segura da artéria lombar após identificação da origem da artéria radicular magna ${ }^{3,9}$. A abordagem cirúrgica aberta envolve ainda a manipulação direta sobre uma parede arterial enfraquecida pelo processo inflamatório 
crônico, com risco de lesão direta e conseqüente sangramento aumentado em uma região de difícil dissecção e controle.

\section{Referências}

1. Halak M, Kligman M, Loberman Z, Eyal E, Karmeli R. Spontaneous ruptured lumbar artery in a chronic renal failure patient. Eur J Vasc Endovasc Surg. 200;21:569-71.

2. Sofocleous CT, Hinrichs CR, Hubbi B, Doddakashi S, Bahramipour P, Schubert J. Embolization of isolated lumbar artery injuries in trauma patients. Cardiovasc Intervent Radiol. 2005;28:730-5.

3. Marty B, Sanchez LA, Wain RA, et al. Endovascular treatment of a ruptured lumbar artery aneurysm: case report and review of the literature. Ann Vasc Surg. 1998;12:379-83.

4. Isokangas JM, Perälä JM. Endovascular embolization of spontaneous retroperitoneal hemorrhage secondary to anticoagulant treatment. Cardiovasc Intervent Radiol. 2004;27:607-611

5. Gutarra F, Rodriguez Asensio J, Miceli M, Mareso E. Ruptured femoropopliteal artery aneurysms in von Recklinghausen neurofibromatosis. J Vasc Surg. 2007;46:808-11.

6. Stevens KJ, Gregson RH, Kerslake RW. False aneurysm of a lumbar artery following vertebral biopsy. Eur Spine J. 1997;6:205-7.
7. Dausse F, Chevallier P, Motamedi JP, Amoretti N, Cua E, Bruneton JN. Lumbar false aneurysms following image-guided interventive procedures for spondylodiskitic abscesses. Skeletal Radiol. 2006;35:949-52.

8. Crook TJ, Whyman MR, Poskitt KR. Lumbar artery aneurysm associated with abdominal aortic aneurysm in a 72-year-old man. Eur J Vasc Endovasc Surg. 2000;20:105-7.

9. Theos A, Korf BR; American College of Physicians; American Physiological Society. Pathophysiology of the neurofibromatosis type I. Ann Intern Med. 2006;144:842.

10. Kipfer B, Lardinois D, Triller J, Carrel T. Embolization of a ruptured intercostal artery aneurysm in type I neurofibromatosis. Eur J Cardiothorac Surg. 2001;19:721-3.

11. Riccardi V. Neurofibromatosis: phenotype, natural history, and pathogenesis. The Johns Hopkins University Press. 1992:124-7.

12. Sclafani SJ, Florence LO, Phillips TF, et al. Lumbar arterial injury: radiologic diagnosis and management. Radiology. 1987;165:709-14.

13. Takase K, Sawamura Y, Igarashi K, et al. Demonstration of the artery of Adamkiewicz at multi- detector row helical CT. Radiology. 2002;223:39-45.

Correspondência:

Wilson de Oliveira Sousa Junior

Rua Capote Valente, 189/1401, Pinheiros

CEP 05409-000 - São Paulo, SP

Tel.: (11) 9449.1364

E-mail: wosj@ig.com.br

\section{O conteúdo do J Vasc Bras está disponivel em português e em inglês no site do Jornal Vascular Brasileiro em www.jvascbr.com.br}

